\title{
Compromise Scheme for Assigning Tasks on a Homogeneous Distributed System
}

\author{
Joo-Man Kim, Member, KIMICS
}

\begin{abstract}
We consider the problem of assigning tasks to homogeneous nodes in the distributed system, so as to minimize the amount of communication, while balancing the processors' loads. This issue can be posed as the graph partitioning problem. Given an undirected graph $G=($ nodes, edges), where nodes represent task modules and edges represent communication, the goal is to divide $n$, the number of processors, as to balance the processors' loads, while minimizing the capacity of edges cut. Since these two optimization criteria conflict each other, one has to make a compromise between them according to the given task type. We propose a new cost function to evaluate static task assignments and a heuristic algorithm to solve the transformed problem, explicitly describing the tradeoff between the two goals. Simulation results show that our approach outperforms an existing representative approach for a variety of task and processing systems.
\end{abstract}

Index Terms - task assignment problem, homogeneous system, load balancing, graph partitioning.

\section{I.INTRODUCTION}

THE cluster systems are not only provide facilities for utilizing resources and/or data at remote sites, but also enhance system performance and reliability with the multiplicity of processors and communication paths. Distributed applications range from large data base installations where the computing load is distributed for organizational efficiency, to small signal-processing systems where computation must be done very fast in a real-time environment.

There are, however, many problems to be resolved before realizing the potential of a distributed system, such as file and task assignment [6]. To alleviate part of these problems, we consider the problem of assigning tasks in cluster computing systems. Specifically, we will deal with static assignment of a given task to the processors in a distributed system which neither requires nor precludes the subsequent dynamic migration of the task. In particular, we are interested in developing centralized task assignment algorithms using global knowledge of the task and system characteristics. These task assignment algorithms attempt to assign each task to the processors so

Manuscript received February 18, 2011; revised April 1, 2011; accepted April 15, 2011

Joo-Man Kim is with the Department of Applied IT and Engineering, Pusan National University, Pusan609-735, Korea (Email: joomkim@pnu.edu) as to achieve such goals as the minimization of interprocessor communication, good load balancing among the processors, a small response time for the task, and efficient utilization of system resources in general. This statement of the static task assignment problem is also useful for assignment of tasks to fewer processors than the distributed algorithm was initially designed for, at the time of initial assignment or dynamically at execution time due to node failure, node withdrawal, or phase transitions in the distributed computation [7].

In this paper, we propose a new cost function for making and evaluating static task assignments, which describes the inherent conflict between the goals of minimizing communication and balancing loads by combining the two criteria into a single objective function. Also, the system designer scanmake an appropriate compromise between the two conflicting goals by systematically adjusting the weight in this cost function according to the underlying task type. We show that the task assignment problem can be modeled as the problem of minimizing the cost of an $n$-cut of a graph (the minimum $n$-cut problem) instead of the minimum balanced $n$-cut problem.

Note that while the minimum balanced $n$-cut problem has two objectives, the minimum $n$-cut problem has only one objective to optimize. The new problem, however, systematically deals with both communication cost minimization and load balancing. The main result of this paper is the development of a heuristic algorithm that allows the system designers to systematically study the effects of relaxing the load balancing constraint on the total communication cost.

The remainder of this paper is organized as follows. The next section presents background information on the task assignment problem. Section IIIdescribes the system model and problem statement for the task assignment problem. It is shown in Section IV that the task assignment problem can be modeled as the problem of finding a minimum $n$-cut in a graph using a graph-modification technique. In Section $\mathrm{V}$, we present an iterative algorithm to solve this problem. Some experimental results and concluding remarks are made in the last two sections.

\section{II.BACKGROUND}

The task assignment problem was first introduced by Stone[2]. Stone's original work lays down the TIG model 
to represent sequentially executing tasks. Bokhari[3][4] conducted a number of task-assignment studies without any inter-task constraints by minimizing total execution and communication costs. Lee[6][8] proposed an exact algorithm that map TIGs to processors in the array networks for minimizing the sum of total execution and communication costs. Lo[7] proposed some heuristic algorithms to improve the degree of concurrency in task assignment by extending Stone's model. Salman[10] proposed a particle swarm optimization for the static task assignment problem to effectively exploit the capabilities of distributed or parallel computing systems. Other researchers[11][12] investigated the task assignment problem by minimizing communication subject to certain constraints on the degree of load balancing, or the minimization of task completion time.

There are numerous studies addressing the task assignment problem under various characterizations. For some later works on mapping TIGs to processors in order to minimize turnaround time see [14] for exact algorithms under processor heterogeneity and network homogeneity; [15] for exact algorithms under processor and network heterogeneity and [13] for heuristics that map TIGs to processors in order to minimize total communication time in a heterogeneous network.

The task assignment problem can be modeled as the problem of partitioning the nodes of a graph into $n$ subsets so as to minimize the cost of the $n$-cut (i.e., the communication cost) and balance the subset size (i.e., load balancing). We will call this problem the minimum balancedn-cut problem which is known to be NPcomplete [6][9].Kernighan and Lin[1] proposeda highlyefficient heuristic for the case of $n=2$. The heuristic can be applied to solve theminimum balanced $n$-cut problem when $n>2$. However, improvements in these heuristics were made for the case of $n=2$, and hence, they have littleuse for the case of $n>2$, because their efficiency decreases significantly as $n$ gets larger [6].

Moreover, they all minimize the cutset cost and consider load balancing only as a constraint. It is very difficult for them to make a tradeoff between load balancing and communication minimization for the following two reasons. First, they do not take into account the degree of load balancing as long as they meet the load balancing constraints. Thus, in case a loose constraint is used, they may yield unbalanced assignments because their communication costs are slightly lower than the costs of other well-balanced assignments. Second, they do not consider any assignment which does not satisfy the load balancing constraint. Therefore, when a strict load balancing constraint is to be met, they may select assignments with much higher communication costs than others due to the constraint. However, for the task assignment problem, both load balancing and communication minimization should be achieved and must be considered at the same time. Also, the system designers should be able to make an appropriate compromise between the two criteria according to the given task type. Thus, the existing heuristics are not very useful for the above task assignment problem.

\section{TASK SYSTEM AND PROBLEM STATEMENT}

\section{A. Task System Model}

We assume that a given distributed system consists of $n$ nodes and an interconnection network that provides full connectivity among the nodes. Formally, we define a task force as a set of $m$ tasks $T=\left\{t_{1}, t_{2}, \cdots, t_{m}\right\}$ which are to be assigned to $n$ nodes $P=\left\{p_{1}, p_{2}, \cdots, p_{n}\right\}$ in the homogeneous distributed system. Let $x_{i}$ be the execution cost of task $i$ and let the interaction among the tasks in $T$ be represented by a TIG, in which nodes correspond to the tasks in $T$ and there exists an edge between two nodes if and only if the corresponding tasks interact. Task assignment to processors, is given by a matrix $a$, where $a_{i p}$ is 1 , if task $i$ is assigned to processor $p$, and 0 otherwise. Let $c_{i j}$ be a binary matrix such that denote the communication cost between two tasks $t_{i}$ and $t_{j}$ if they are assigned to different processors. Obviously, if no edge exists between $t_{i}$ and $t_{j}$ in the graph, then $c_{i j}=0$. Both the execution and communication costs are derived from the types of the tasks. A task set $T$ can be one of two types: computation module or communication module. They may be specified explicitly by the programmer, or deduced automatically by the compiler, or refined by monitoring the previous executions of the task. In this paper, we presume that the data about the execution and communication costs are available and they are positive integer values.
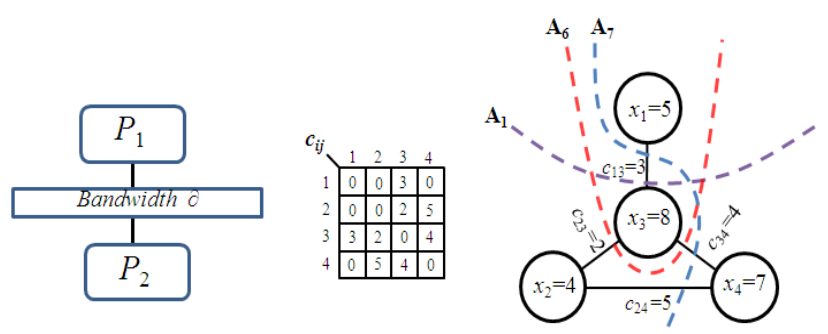

Fig. 1. TIG for a 2-processor system.

For tractability we ignore other attributes of the taskprocessor system such as timing and precedence constraints, and we assume that the communication costs are independent of the communication link upon which they occur. We will assume that the communication cost between two tasks executed on the same processor is negligible, since such communication is accomplished by 
accessing local shared memory, as opposed to using interprocessor communication links. An assignment of tasks to processors can formally be described by a function from the set of tasks to the set of processors, $A: T \rightarrow P$. In a system of $\boldsymbol{m}$ tasks and $\boldsymbol{n}$ homogeneous processors there are $1 / 2 \cdot n^{m}$ possible assignments of tasks to processors. For example, consider the TIG for two-processor system depicted in Figure 1.

To minimize the communication overhead, assignment $A_{1}$ with the communication cost3is the best solution where $t_{1}$ is assigned to $P_{1}$ and all the other tasks assigned to $P_{2}$. Assignment $A_{1}$ however, induces the processor 'load6 and 22 on $P_{1}$ and $P_{2}$, respectively, making the system highly-unbalanced. To achieve load balancing, we can choose assignment $A_{7}$ that is perfectly load-balanced. However, the communication cost of $A_{7}$ is 12 which is much higher than that of $A_{1}$. If $t_{4}$ assigned to $P_{1}$ is exchanged $t_{3}$ assigned to $P_{2}$ under $A_{7}$.i.e., assignment $A_{6}$, the Comm. cost decreases to 6 at a small loss of load balancing. Assignment $A_{6}$ may be chosen if no strict condition is imposed on the load balancing objective. Therefore, the system designer should be able to make an appropriate compromise between the two conflicting objectives according to the task type.

\section{B. Problem Statement}

The communication cost of an assignment $A$, denoted as $\operatorname{Comm}(A)$, is then calculated as :

$$
\operatorname{Comm}(A)=\sum_{i<j} \sum_{p=1}^{n} a_{i p}\left(1-a_{j p}\right) c_{i j}
$$

The load $L_{p}$ of processor $p$ is the total cost of running the tasks assigned to processor $p$ under this assignment:

$$
\begin{aligned}
& L_{p}=\sum_{i=1}^{m} a_{i p} x_{i} \\
& L_{\text {tot }}=\sum_{p=1}^{n} L_{p}
\end{aligned}
$$

The standard deviations is essential for assessing the degree of dispersion of the loads around its mean load. If this load could be uniformly distributed among the $n$ processors, each processor would be assigned a share of mean $\bar{L}$. For a given task, $L_{\text {tot }}$ and $\bar{L}$ are uniquely determined irrespective of the assignment of the task. $\sigma^{2}(A)$, the variance, for each assignment $A$,

$\sigma^{2}(A)=\sum_{p=1}^{n} \frac{\left(L_{p}-\bar{L}\right)^{2}}{n}=\frac{n-1}{n^{2}} L_{t o t}{ }^{2}-\frac{2}{n} \sum_{p<q} L_{p} \cdot L_{q} . \quad$ is $\quad$ the variance of load distribution under the assignment. The first term, $(n-1) L_{t o t}{ }^{2} / n^{2}$, is constant for a given task, and only the second term, $(2 / n) \sum_{p<q} L_{p} \cdot L_{q}$, varies between 0 and $(n-1) L_{t o t}{ }^{2} / n^{2}$, depending on the assignment of the task. Then, the following inequality holds :

$$
0 \leq \sigma^{2}(A) \leq \frac{(n-1)}{n^{2}} L_{\text {tot }}{ }^{2}, \text { for all assignments } A .
$$

The variance of load distribution indicates how evenly the load is distributed among the processors, i.e., the lower the variance the better balanced the load distribution. The maximum value of $\sigma^{2}(\cdot)$ is achieved when all the tasks are assigned to a single processor. $\sigma^{2}(\cdot)$ reaches its minimum value when all the processors are most evenly loaded. In such a case, the system is said to be best loadbalanced. Thus, one may use $\sigma^{2}(A)$ as a yardstick to measure the degree of load balancing of each assignment $A$. For simplicity, we define thedegree of load balancing of $A$, denoted as $L B(A)$, as :

$$
L B(A)=1-\frac{\sigma^{2}(A)}{n-1 / n^{2} \cdot L_{t o t}{ }^{2}}=\frac{2 n}{(n-1) L_{t o t}{ }^{2}} \sum_{p<q} L_{p} \cdot L_{q} .
$$

Note that $L B(A)$ always satisfies the inequality $0 \leq L B(A) \leq 1$.Using $\operatorname{Comm}(\cdot)$ and $\sigma^{2}(\cdot)$, we propose the

\begin{tabular}{|c|c|c|c|c|c|c|c|c|}
\hline \multirow[b]{2}{*}{ Assign } & \multicolumn{2}{|c|}{ Assign to Proc. } & \multicolumn{2}{|c|}{ Load } & \multirow[b]{2}{*}{$\sigma^{-}(A)$} & \multirow[b]{2}{*}{$L B(A)$} & \multirow[b]{2}{*}{ Comm } & \multirow[b]{2}{*}{$\cos \Omega(A)$} \\
\hline & $\mathrm{P}_{1}$ & $\mathrm{P}_{2}$ & $\overline{L_{P}}$ & $L_{p}$ & & & & \\
\hline$A_{1}$ & $t_{1}$ & $t_{2} t_{3} t_{4}$ & 5 & 19 & 49 & 0.66 & 3 & $3+\alpha-48.96$ \\
\hline$\overline{A_{2}}$ & $t_{2}$ & $t_{1} t_{3} t_{4}$ & 4 & 20 & 64 & 0.56 & 7 & $7+\alpha \cdot 63.36$ \\
\hline $\mathrm{A}_{3}$ & $t_{3}$ & $t_{1} \quad t_{2} t_{4}$ & 8 & 16 & 16 & 0.89 & 9 & $9+\alpha \cdot 15.84$ \\
\hline $\mathrm{A}_{4}$ & $t_{4}$ & $\begin{array}{lll}t_{1} & t_{2} & t_{3}\end{array}$ & 7 & 17 & 25 & 0.83 & 9 & $9+\alpha \cdot 24.48$ \\
\hline $\mathrm{A}_{5}$ & $\begin{array}{ll}t_{1} & t_{2}\end{array}$ & $t_{3} t_{4}$ & 9 & 15 & 9 & 0.94 & 10 & $10+\alpha \cdot 8.64$ \\
\hline$A_{6}$ & $\begin{array}{ll}t_{1} & t_{3} \\
\end{array}$ & $t_{2} t_{4}$ & 13 & 11 & 1 & 0.99 & 6 & $6+\alpha \cdot 1.44$ \\
\hline $\mathrm{A}_{7}$ & $t_{1} t_{4}$ & $t_{2} t_{3}$ & 12 & 12 & 0 & 1.00 & 12 & $12+\alpha \cdot 0$ \\
\hline$A_{8}$ & $t_{1} t_{2} t_{3} t_{4}$ & $\phi$ & 28 & 0 & 144 & $\infty$ & 0 & $0+\alpha \cdot \alpha$ \\
\hline
\end{tabular}
following cost function :

$$
\begin{aligned}
\operatorname{COST}(A) & =\operatorname{Comm}(A)+\alpha \cdot \sigma^{2}(A) \\
& =\operatorname{Comm}(A)+\alpha \cdot(1-L B(A)) \frac{n-1}{n^{2}} L_{t o t}{ }^{2},
\end{aligned}
$$

TABLE I

\section{ASSIGNMENT RESULT FOR TIG OF FIGURE 1}

The result given in Table 1 are 8-assignments of 4 tasks to 2 processors. where the weighting factor $\alpha$ is some nonnegative constant to be chosen by the system designer. A good compromise between the two criteria can be made by setting $\alpha$ to an appropriate value. For example, if we want to put more emphasis on load balancing, we may set $\alpha$ to a higher value. By setting $\alpha$ to a lower value, one can put more emphasis on communication cost than on load balancing.

\section{B. The Weighting Factor}

Based on the proposed cost function one can represent the tradeoff between load balancing a communication cost by setting $\alpha$ to an appropriate value. There are two extreme cases in the task assignment problem. The one is to assign tasks with the objective of minimizing the communication cost only, without considering load balancing at all. The other is to assign tasks so as to achieve the maximum degree of load balancing. These two extreme cases may occur when one needs to assign highly communication-bound tasks and 
highly computation-bound tasks, respectively. The proposed cost function can represent the objective of the first case by setting $\alpha$ to zero. In the second case, however, we have to estimate a certain value of $\alpha$ at which the maximum degree of load balancing (or best load-balancing) is achieved.

As $\alpha$ increases, the load-balancing degree of an optimal assignment increases until it becomes best loadbalanced. Let $\hat{\alpha}$ be the smallest weighting factor with which optimal assignment are best load-balanced. We call $\hat{\alpha}$ the "upper-bound" of the weighting factor. Then, let $A_{o}^{\hat{\alpha}}$ be an optimal assignment with the weighting factor $\hat{\alpha}$

Definition 1: The gain in communication cost of an assignment $A$, denoted by $\operatorname{Comm}_{\text {gain }}(A)$, is defined as

$$
\operatorname{Comm}_{\text {gain }}(A)=1-\frac{\operatorname{Comm}(A)}{\operatorname{Comm}\left(A_{o}^{\hat{\alpha}}\right)} \text {. }
$$

Definition 2: The loss in load balancing of an assignment $A$, denoted by $L B_{\text {loss }}(A)$, is defined as

$$
L B_{\text {loss }}(A)=1-L B(A) \text {. }
$$

By setting $\alpha$ lower than $\hat{\alpha}$, we gain in communication cost but we lose in load balanced. To make a compromise between the two, we have to determine the weight on each of them according to the given task type. This compromise factor should be reflected in the cost function. Definition3 :A compromise factor $\delta$ is valid if it satisfies the following two conditions :

$$
\begin{aligned}
& \text { if } \operatorname{Comm}_{\text {gain }}\left(A_{1}\right)-\delta \cdot L B_{\text {loss }}\left(A_{1}\right)>\operatorname{Comm}_{\text {gain }}\left(A_{2}\right)-\delta \cdot L B_{\text {loss }}\left(A_{2}\right) \text {, } \\
& \text { then } \operatorname{COST}\left(A_{1}\right)<\operatorname{COST}\left(A_{2}\right) \\
& \text { if } \operatorname{Comm}_{\text {gain }}\left(A_{1}\right)-\delta \cdot \operatorname{LB} B_{\text {loss }}\left(A_{1}\right)=\operatorname{Comm}_{\text {gain }}\left(A_{2}\right)-\delta \cdot L B_{\text {loss }}\left(A_{2}\right) \text {, } \\
& \text { then } \operatorname{COST}\left(A_{1}\right)=\operatorname{COST}\left(A_{2}\right) \text {. }
\end{aligned}
$$

Theorem 1 : The cost function proposed in this paper is a $\delta$-policy if

$$
\alpha=\delta \cdot \frac{\operatorname{Comm}\left(A_{o}^{\hat{\alpha}}\right)}{\frac{N-1}{N^{2}} L^{2}} .
$$

Proof: For any assignments $A_{1}$ and $A_{2}$, suppose $\operatorname{Comm}_{\text {gain }}\left(A_{1}\right)-\delta \cdot L B_{\text {loss }}\left(A_{1}\right)>\operatorname{Comm}_{\text {gain }}\left(A_{2}\right)-\delta \cdot L B_{\text {loss }}\left(A_{2}\right)$.

Then,

$$
\begin{aligned}
& \operatorname{COST}\left(A_{1}\right)-\operatorname{COST}\left(A_{2}\right) \\
& =\operatorname{Comm}\left(A_{1}\right)-\operatorname{Comm}\left(A_{2}\right)+\delta \cdot \operatorname{Comm}\left(A_{o}^{\hat{\alpha}}\right) \cdot\left(\operatorname{LB}\left(A_{2}\right)-\operatorname{LB}\left(A_{1}\right)\right) \\
& =\operatorname{Comm}\left(A_{o}^{\hat{\alpha}}\right)\left(\left(\operatorname{Comm}_{\text {gain }}\left(A_{2}\right)-\operatorname{Comm}_{\text {gain }}\left(A_{1}\right)\right)\right. \\
& \left.\quad+\delta \cdot\left(L B_{\text {loss }}\left(A_{1}\right)-L B_{\text {loss }}\left(A_{2}\right)\right)\right) \\
& \\
& \quad 0 .
\end{aligned}
$$

Obviously, $\operatorname{COST}\left(A_{1}\right)=\operatorname{COST}\left(A_{2}\right)$ from definition 3.

Therefore, it is a valid weighting factor.

To further investigate the effects of compromise factor $\delta$ on both load balancing and communication cost, 20 experiments were performed. In these experiments, 30 $\leq m \leq 50$ (the number of tasks), and $2 \leq n \leq 4$ (the number of processors) were picked randomly. An edge was added between any two nodes with the probability $p_{d}$. The weight of each edge and the size of each node were picked randomly between $0 \& r_{w}$ and $0 \& r_{s}$, respectively. Also, in each experiment, $p_{d}, r_{w}$, and $r_{s} w e r e$ chosen randomly between $20 \& 40,1 \& 10$, and $5 \& 15$, respectively.

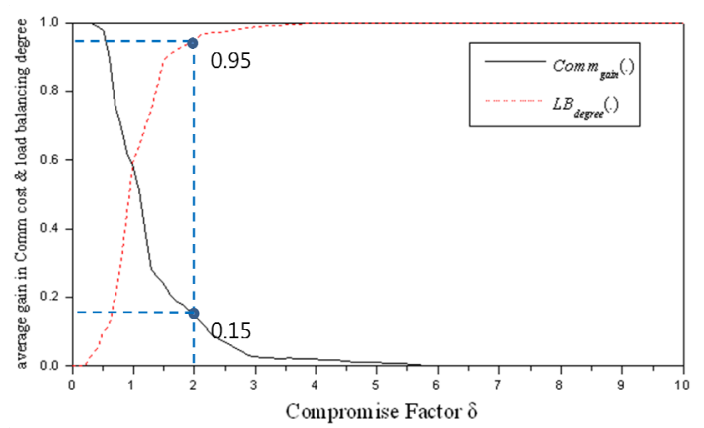

Fig. 2. Average $\operatorname{Comm}_{\text {gain }}(\cdot)$ and $L B(\cdot)$ by compromise factor $\delta$.

Figure.2 shows the average gain in communication cost, $\operatorname{Comm}_{\text {gain }}(\cdot)$, and the average load-balancing degree, $L B(\cdot)$, of optimalassignment, as the value of $\delta$ increases from 0 to 10 . As $\delta$ increases, $\operatorname{Comm}_{\text {gain }}(\cdot)$ decreases while $L B(\cdot)$ increases, as expected. As shown in this figure, when $\delta=2$ we can obtain, on average, the gain of 0.15 in communication with the loss of 0.05 in load balancing.

\section{IV.PROBLEM TRANSFORMATION}

An $n$-cut of a graph $G$ is a set of edges that partitions the nodes of $G$ into $n$ disjoint subsets $P_{1}, P_{2}, \cdots, P_{n}$. The weight of an $n$-cut is the sum of the weights of the edges in the $n$-cut. The size of each subset $P_{k}$ is the sum of the sizes of nodes in $P_{k}$. There is a one-to-one correspondence between $n$-cuts of the TIG of a given task and assignments of the task to $n$ processors. Then, the weight of an $n$-cut is equal to the communication cost and the load-balancing degree of size distribution among the $n$ disjoint subsets is equal to the load-balancing degree of the corresponding assignment. Therefore, the task assignment problem considered here is equivalent to the problem of finding an $n$-cut of a graph with the objective of minimizing the $n$ cut weight as well as balancing the size of subset. In the original TIG, each cutest has no information on the size of the resulting subsets. Thus, it is difficult to develop efficient heuristics on the original graph, since one must consider the load-balancing degree of an existing partition as well as the cutest weight of the partition. KernighanLin's(K\&L) algorithm, for example, keeps an existing partition balanced by using pairwise-exchange of nodes of the same size given an initial balanced partition and tries to minimize the cutest weight. Thus, we can see that, in $\mathrm{K} \& \mathrm{~L}$ algorithm, there exist two separate parts which consider communication and load balancing, respectively. If we modify the TIG such that the weight of each edge 
contains the information on the size (i.e., running costs) of the corresponding nodes as well as the original weight of the edge, it gets easy for us to develop an efficient method for finding a tradeoff between the two objectives because both pieces of information are included in the weights of the edges in an $n$-cut after modification. We propose a modification technique for the TIG of a given task such that any cutest on the modified graph, say $G$, has the information on the load-balancing degree of the corresponding assignment as well as the original weights of the edges. For any two nodes $m_{i}$ and $m_{j}$ of the TIG :

1. if there is an edge between them then modify the weight of the edge to be $W_{i j}-x_{i} \cdot x_{j} \cdot(2 \alpha / n)$

2. otherwise, make an edge between them which has the weight of $--x_{i} \cdot x_{j} \cdot(2 \alpha / n)$, where $\alpha=\delta \cdot \operatorname{Comm}\left(A_{o}^{\hat{\alpha}}\right) /\left(\frac{N-1}{N^{2}} L_{t o t}{ }^{2}\right)$.

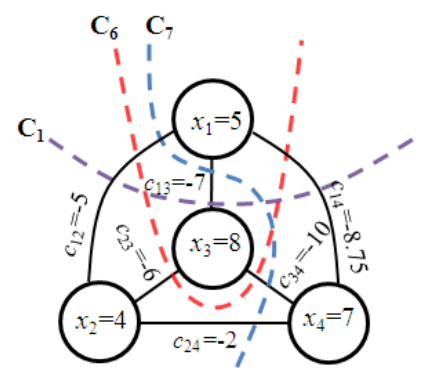

Fig. 3. An example of graph modification.

For example, for the TIG in Figure 1, the modified graph $G$ with a compromise factor $\delta=3$ is shown in Figure 3. Note that, in this example, $\operatorname{Comm}\left(A_{o}^{\hat{\alpha}}\right)=12$, i.e.,

$$
\begin{aligned}
\alpha & =\delta \cdot \operatorname{Comm}\left(A_{o}^{\hat{\alpha}}\right) /\left(\frac{N-1}{N^{2}} L_{\text {tot }}{ }^{2}\right) \\
& =3 \times 12 /\left(\frac{2-1}{2^{2}} 24^{2}\right) \\
& =0.25
\end{aligned}
$$

The 2-cuts $C_{1}, C_{6}, C_{7}$ in Figure 3 correspond to the assignments $A_{1}, A_{6}, A_{7}$ in Figure 1 , respectively. An algorithm based on this transformation requires more storage since the initial potentially-sparse graph is transformed into an almost complete graph before the algorithm begins. However, with the resulting modified graph $G$, it becomes much easier to devise efficient heuristics for the task assignment problem since each edge has the information on both the execution and the communication costs. Since each $n$-cut on the modified graph $G$ has the complete information on the total cost of its corresponding assignment, the task assignment problem considered here can be transformed into the problem of finding a minimum-weight $n$-cut in a graph which is called the minimumn-cut problem. This is proved in Theorem 4.

Theorem 2 : The task assignment problem can be transformed into the minimum $n$-cut problem.

Proof : Let an $n$-cut $C$ of a modified graph $G$ correspond to an assignment $A$. Suppose $C$ partitions the nodes of $G$ into $n$ subsets $P_{1}, P_{2}, \cdots, P_{n}$. Let each subset $P_{k}$ be $\left\{m_{k_{i}}: 1 \leq i \leq p_{k}\right\}$. Then the weight of $C, W(C)$, is as follows.

$$
\begin{aligned}
W(C) & =\sum_{k<l}\left(\sum_{\substack{1 \leq i \leq p_{k} \\
1 \leq j \leq p_{l}}}\left(W_{k_{i} l_{j}}-x_{k_{i}} \cdot x_{l_{j}} \cdot \frac{2 \alpha}{n}\right)\right) \\
& =\sum_{k<l} \sum_{\substack{1 \leq i \leq p_{k} \\
1 \leq j \leq p_{l}}} W_{k_{i} l_{j}}-\left(\sum_{k<l} L_{k} \cdot L_{l}\right) \cdot \frac{2 \alpha}{n} \\
& =\operatorname{Comm}(A)-\left(\sum_{k<l} L_{k} \cdot L_{l}\right) \cdot \frac{2}{n} \cdot \alpha \\
& =\operatorname{COST}(A)-\frac{n-1}{n^{2}} L_{t o t}{ }^{2} \cdot \alpha .
\end{aligned}
$$

The second term of the last equation is constant for each task. Therefore, the task assignment problem is equivalent to the problem of finding a minimum-cost $n$ cut in a graph generated by the proposed graph modification technique. For example, the weights of $C_{1}, C_{6}, C_{7}$, in Figure 3 are $-20.75,-29.75$, and -12.0 , respectively. $C_{6}$ is the minimum weight 2 -cut. Then the costs of their corresponding assignments are :

$$
\begin{aligned}
& \operatorname{COST}\left(A_{1}\right)=W\left(C_{1}\right)+\frac{n-1}{n^{2}} L_{t o t}{ }^{2} \cdot \alpha=-20.75+144 \times 0.25=15.25, \\
& \operatorname{COST}\left(A_{6}\right)=W\left(C_{6}\right)+\frac{n-1}{n^{2}} L_{t o t}{ }^{2} \cdot \alpha=-29.75+144 \times 0.25=6.25, \\
& \operatorname{COST}\left(A_{7}\right)=W\left(C_{7}\right)+\frac{n-1}{n^{2}} L_{t o t}{ }^{2} \cdot \alpha=-24.0+144 \times 0.25=12 .
\end{aligned}
$$

Therefore, as shown in Figure 1 , when $\delta=3$, the best assignment is $A_{6}$ which corresponds to the minimum weight 2-cut $C_{6}$.

\section{ASSIGNMENT ALGORITHM AND ANALYSIS}

\section{A. The minimum n-cut problem}

When the value of $\delta$ is given, we have to find $A_{o}^{\hat{\alpha}}$ so as to determine the weighting factor $\alpha$. Since it is computationally intractable to find such an optimal best load-balanced assignment, we have to resort to heuristics. This problem can be transformed to the minimum $n$-cut problem using the proposed graph modification technique by setting $\alpha$ to its upper bound $\hat{\alpha}=\max _{i}\left(\sum_{j=1}^{n} n \cdot W_{i, j} / 4 x_{i}\right)$.We first present a heuristic algorithm for the minimum $n$-cut problem. We wish to find a minimum weight $n$-cut $C_{o}$ which assigns the nodes in $V$ to $n$ subsets (i.e., processors) $P_{1}, P_{2}, \cdots, P_{n}$. 
Here, $\quad W\left(C_{o}\right)=\min _{C} W(C)=\min _{C} \sum_{i, j} c_{i, j}$, where $t_{i}$ and $t_{j}$ are assigned different subsets. This is called the minimum n-cut problem.

The basic approach for the minimum n-cut problem is to start with an arbitrary assignment and to improve it by iteratively choosing one node in a processor and moving it to another processor. The node to be moved is chosen such that a maximum decrease in the cutest weight may be obtained (or minimum increase if no decrease is feasible). The algorithm consists of a series of passes (iterations): in each pass, every node should be moved only once. In each pass, the modes to be moved are chosen among those that have not yet been moved during the pass. The $m$ assignments produced during the pass are examined and the one with the smallest cutest is chosen as the starting assignment for the next pass. Passes continue until no further improvement in the cutest weight can be made.

Definition 4: We define the gain $g\left(t_{a}, P_{j}\right)$ of each node $t_{a}$ in $P_{i}$ over $P_{j}(i \neq j)$ as the amount by which the cutset weight would decrease if $t_{a}$ is moved from $P_{i}$ to $P_{j}$, i.e.,a node with the largest gain is $\mathrm{s}$ elected as the node to be moved.

For example, if the gain of $t_{a}$ in $P_{i}$ over $P_{j} g\left(t_{a}, P_{j}\right)$, is maximum, $t_{a}$ will be moved from $P_{i}$ to $P_{j}$, one-move operation. It will often be the case that the gain is nonpositive. In that case, we still move the node with the expectation that the move will allow the algorithm to "climb out of local minima" as was done by Kernighan and Lin [1]. After all nodes have been forced to move, the best partition encountered during the pass is taken as the output of the pass.

To prevent the one-move process from "thrashing" or going into an infinite loop, we immediately "lock" each selected node in its new set for the remaining part of a pass. Thus, only "free" nodes are actually allowed to make one-move during the pass until all nodes are locked. After each pass, all nodes are released and hence become "free" again. After moving the selected node, we recalculate the gains of all the other free nodes. For example, if $t_{r}$ in $P_{i}$ is moved to $P_{j}$, the new gains of free nodes are as follows:

$$
\begin{aligned}
& \hat{g}\left(t_{a}, P_{j}\right)=g\left(t_{a}, P_{j}\right)+2 c_{a r}, \text { for all } t_{a} \in P_{i} \\
& \hat{g}\left(t_{a}, P_{l}\right)=g\left(t_{a}, P_{l}\right)+c_{a r}, \text { for all } t_{a} \in P_{i}, 1 \leq l(\neq i, j) \leq n, \\
& \hat{g}\left(t_{b}, P_{i}\right)=g\left(t_{b}, P_{i}\right)-2 c_{b r}, \text { for all } t_{b} \in P_{j}, \\
& \hat{g}\left(t_{b}, P_{l}\right)=g\left(t_{b}, P_{l}\right)-c_{b r}, \text { for all } t_{b} \in P_{j}, 1 \leq l(\neq i, j) \leq n, \\
& \hat{g}\left(t_{c}, P_{i}\right)=g\left(t_{c}, P_{i}\right)-c_{c r}, \text { for all } t_{c} \in P_{l}, 1 \leq l(\neq i, j) \leq n, \\
& \hat{g}\left(t_{c}, P_{j}\right)=g\left(t_{c}, P_{j}\right)+c_{c r}, \text { for all } t_{c} \in P_{l}, 1 \leq l(\neq i, j) \leq n .
\end{aligned}
$$

The correctness of these expressions is easy to verify.

\section{B. The minimum $n$ - cut algorithm}

In the first step, compute the gains for all nodes of vertices for an initial assignment. This assignment may be obtained simply by assigning all the nodes randomly. Set all the nodes "free". In the second step, choose a free $t_{a}$ in $P_{i}$ such that

$$
g_{1}=g\left(t_{a}, P_{j}\right)=\sum_{t_{b} \in P_{j}} c_{a b}-\sum_{t_{c} \in P_{i}} c_{a c}
$$

is maximum; and move $t_{a}$ from $P_{i}$ to $P_{j}$ and lock it. For notational convenience, mark $\left(t_{a}, P_{i}\right)$ as $\left(t_{1}^{\prime}, P_{1}^{\prime}\right)$. In the third step, recalculate the gains for all free nodes.

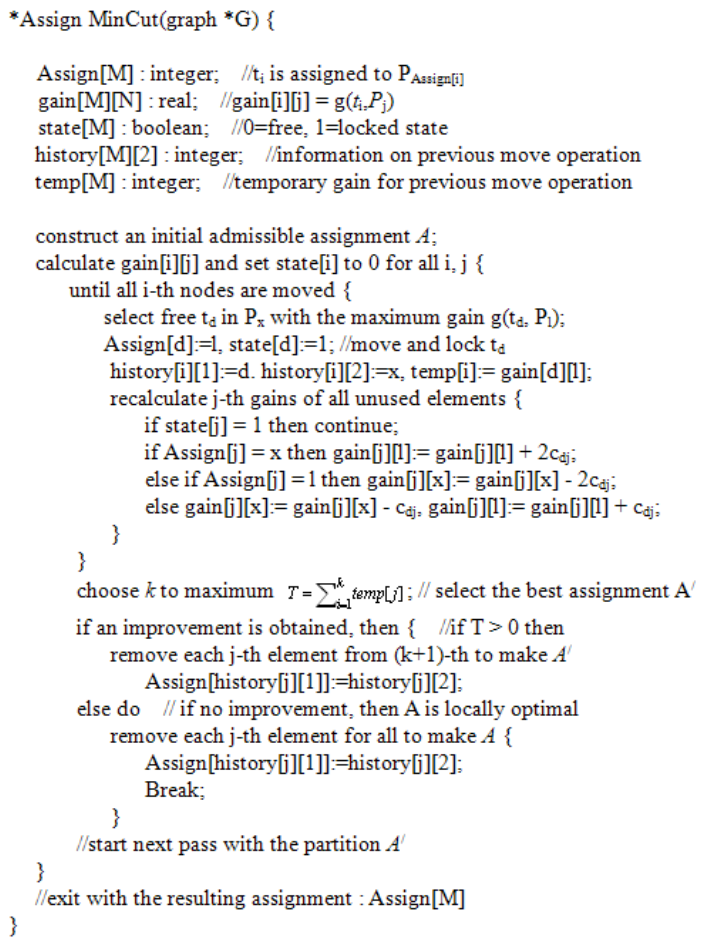

Fig. 4. The minimum $n$-cut algorithm:MinCut.

Now, repeat the second step, choosing a free node $t_{2}^{\prime}$ in $P_{2}^{\prime}$ with the maximum gain of $g_{2}$. Thus, $g_{2}$ is the additional gain when node $t_{2}^{\prime}$ (as well as $t_{1}^{\prime}$ ) is moved, and this additional gain is maximum, given the previous choices. Move $t_{2}^{\prime}$ to the corresponding set and lock it. Continue this process until all free nodes have been exhausted, thus identifying $\left(t_{3}^{\prime}, P_{3}^{\prime}\right), \ldots,\left(t_{m}^{\prime}, P_{m}^{\prime}\right)$, and the corresponding maximum gains $g_{3}, \ldots, g_{m}$.If $K=$ $\left\{t_{1}^{\prime}, t_{2}^{\prime}, \cdots, t_{k}^{\prime}\right\}$, then the decrease in weight when the nodes in the set $K$ are moved is precisely $g_{1}+g_{2}+\ldots+g_{k}$. Note that some of the $g_{i}$ 's may be negative.

Choose $k$ that maximizes the partial sum $\sum_{i=1}^{k} g_{i}=T$. Now, if $T>0$, a reduction in $T$ can be made by moving the nodes in $K$. After this is done, the whole procedure is repeated from the first step with the resulting 
assignment as the initial assignment. If $T \leq 0$, we have arrived at a local optimum assignment. We now have the choice of repeating with another starting assignment. The algorithm described thus far will be called MinCut, which is formally described in a Pascal-like algorithm in Fig. 4. Especially, an M-tuple implemented in the form of an array Assign[M] is used to describe the current assignment. The $\mathrm{k}$-thelement of the array gives the processor number to which task $t_{k}$ is assigned under the current assignment. History $[\mathrm{M}][2]$ is used to save the history of one-move operations. For example, when onemove on $t_{k}$ is made from $P_{i}$ to $P_{j}$ history $[\mathrm{k}][1]=j$ and history $[\mathrm{k}][2]=i$.

\section{Time-Complexity Analysis}

For time complexity analysis, we define a pass to be the operation involved in making one cycle from step 2 to step 5 of $\mathrm{MinCut}$. The computing time needed for step 2 is $O\left(N M^{2}\right)$ since we need an $O(M)$ time to compute the gain of each node over each processor. Each iteration of step 3 needs an $O(N M)$ computing time due mainly to the selection of a node with the largest gain. Thus, the total time needed for step 3 is $O\left(N M^{2}\right)$. The computing time of $O(M)$ is sufficient for step 4 and 5 . Therefore, the total computing time for a pass is $O\left(N M^{2}\right)$.

The number of passes required for $\mathrm{MinCut}$ to terminate is small. In our experiments on graphs with up to 200 nodes and with various values of $N$ (up to 20), it has almost always been between 3 and 6. From these experiments, we can see that the number of passes does not strongly depend on the value of $M$.

\section{The Assignment Algorithm}

To determine the weighting factor a, we have to find $A_{o}^{\hat{\alpha}}$ which corresponds to a minimum $n$-cut of the graph $G^{\prime}$ resulting from the modification of the original TIG with $\alpha=\hat{\alpha}$. After determining $\alpha$, the final assignment can be found on the graph $G$ modified from TIG with the value of $\alpha$ determined. Therefore, we have to run MinCutexactly twice to find the final assignment. The assignment algorithm TaskAssign is shown in Fig. 7.

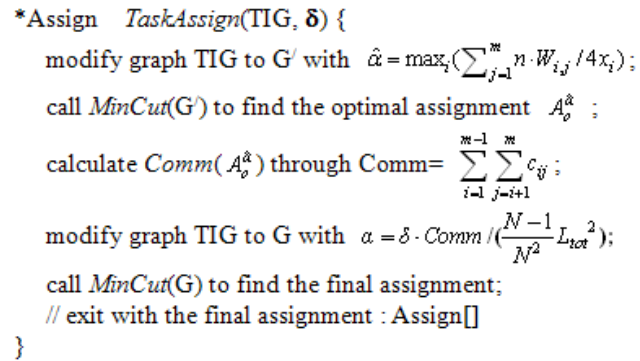

Fig. 5. The optimal task assignment : TaskAssign.
To determine the weighting factor $\alpha$, we have to find $A_{o}^{\hat{\alpha}}$ which corresponds to a minimum $n$-cut of the graph $G^{\prime}$ resulting from the modification of the original TIG with $\alpha=\hat{\alpha}$. After determining $\alpha$, the final assignment can be found on the graph $G$ modified from TIG with the value of $\alpha$ determined. Therefore, we have to run MinCutexactly twice to find the final assignment. The assignment algorithm TaskAssign is shown in Figure 5.

\section{EXPERIMENTAL RESULT AND ANALYSIS}

A number of experiments were performed to evaluate the performance of $\mathrm{MinCut}$. The experiments were performed for the task assignment problem with the constraint of perfectload balancing, i.e., to assign tasks so as to minimize communication cost while maintaining perfect loadbalancing. This problem is equivalent to the problem of perfectly balanced $N$-way partitioning of graphs [5]. On the basis of the experimental evidence as previously described, $\alpha$ is set to its approximate upper bound.

We have performed a number of experiments for regular and random graphs. For regular, we performed two experiments for the cases of $10 \times 10$ two-dimensional meshes and full binary trees of depth 7 , respectively, to compare our algorithm with K\&L for the balanced twocut problem. For each experiment,20 graphs were tested. Also, the experiments in case of $N=4$ were performed for random graphs to compare our heuristic algorithm with $\mathrm{K} \& \mathrm{~L}$ algorithm adapted to the general balanced $n$-cut problem.

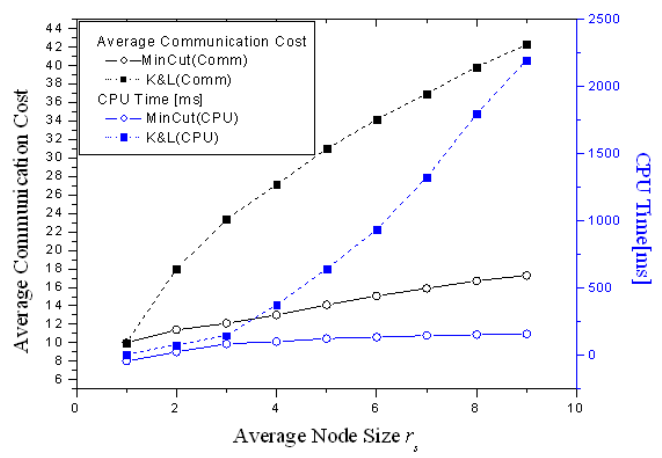

Fig. $6.10 \times 10$ 2DMesh: Regular TIG.

The size of each node was picked randomly between 0 and $r_{s}$. Each experiment is divided into nine cases: $r_{s}=$ $1,2, \ldots, 9$. For all generated graphs, the weight of each edge was set to one in order or evaluate the performance in terms of the number of edges in the final cutest.

On all graphs tested, both K\&L and our algorithms always found perfectly balanced partitions. The main performance measure is therefore the cost of the resulting cutsets, i.e., the communication cost. 
The experimental results for meshes and trees are summarized in Figure 6 and Figure 7. For each experiment, both $\mathrm{K} \& \mathrm{~L}$ and our algorithm always found optimal solutions when $r_{s}=1$.However, as can be seen in the figure, our algorithm significantly outperforms $K \& L$ as the size difference among the nodes increases.

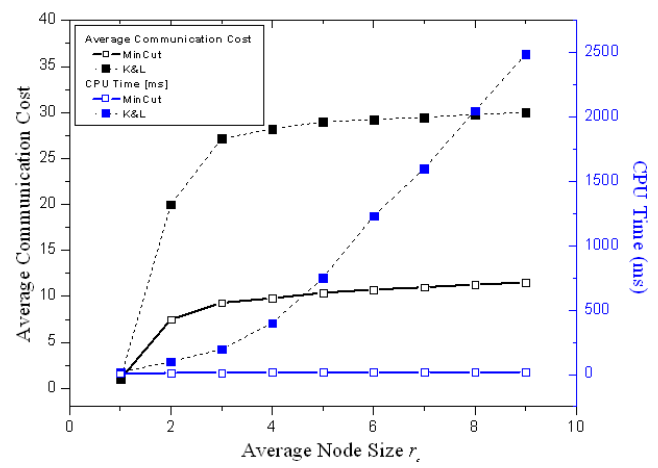

Fig. 7. depth-7 Tree Regular TIG.

For random, our experiment was performed for the task assignment problem with two conflicting objectives, i.e., minimizing communication cost and balancing loads. To compare our "integrated" approach with an existing approach in which minimizing communication cost is the only optimization criterion and a balancing condition is given as a constraint, we modified the "pair wiseexchange" scheme of K\&L algorithm as: any two different-size nodes can be candidates to be exchanged if the balancing constraint is satisfied after exchanging them.

The experiments for $N=4$ were performed for random graphs. We compared the solution quality of our MinCut with that of modified K\&L for compromise factor $\delta(0$ to 10). For the experiment, 100 random graphs were generated with 100 nodes and about 1500edges where the weight of each edge was set to one.

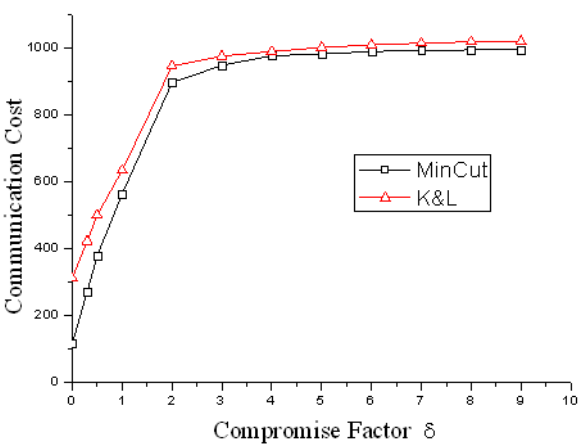

Fig. 8. Comm. Cost Comparison by $\boldsymbol{\delta}$.

The experimental results for random graphs are summarized in Figure 8 and Figure 9. Our algorithm is shown to outperform K\&L in solution quality even when $r_{s}=3$. Also, the time consumed by our algorithm is less than that in K\&L.
As shown in these figures, the average communication cost for MinCut is much less than that for K\&L. The performancegap becomes large as the balancing constraint becomes loose, i.e., as $\delta$ decreases.

From these results of the experiment, we can see that our integrated approach is better than the existing approach for the task assignment problem considered in this paper.

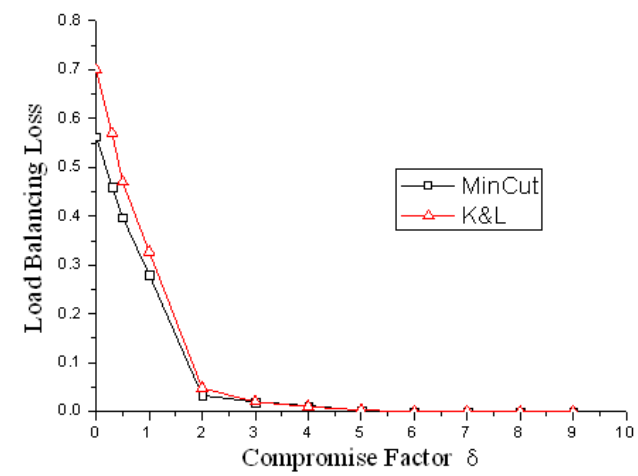

Fig. 9. Load Balancing Loss by $\boldsymbol{\delta}$.

\section{CONCLUSION}

Our investigation of the static task assignment problem has resulted in the development of an "integrated" approach which systematically resolves the two conflicting goals, i.e., minimizing communication costs and balancing loads. This was done by using the variance statistics of load distribution to represent the degree of load balancing among processors. We proposed a new cost function which represents the inherent tradeoff between the two goals by combining them into a single objective function. We also proposed the weighting and compromise factors with which one can systematically make a compromise between the two conflicting goals according to the underlying task type. The task assignment problem has been shown to be transformed into the minimum $n$-cut problem which has only one objective, whereas the original problem has two objectives to optimize. Also, we have developed a heuristic algorithm for the transformed problem. Experimental results indicate that the algorithm performs quite well on a variety of task-processor systems.

Task assignment still poses to offer a variety of challenging problems such as assignment with resource relocation, real-time constraints, communication link loads, heterogeneous multi-processors, not to mention the precedence relationships and load balancing. While much work has been done dealing with each of these problems, it is interesting to extend our results by increasing the complexity of the model to include such factors 


\section{REFERENCES}

[1] B. W. Kernighan and S. Lin, "An efficient heuristic procedure for partitioning graphs," Bell Syst. Tech. J., vol. 49, pp. 291-307, Feb. 1970.

[2] H. S. Stone, "Critical load factors in distributed computer systems," IEEE Trans. Software Eng., vol. SE-4, pp. 254-258, May 1978.

[3] S. H. Bokhari, "A shortest tree algorithm for optimal assignments across space and time in a distributed processor systems," IEEE Trans. Software Eng., vol. SE-7, pp. 583-589, Nov. 1981.

[4] S. H. Bokhari, "Assignment Problems in Parallel and Distributed Computing," Kluwer Academic Publishers, Boston, 1987.

[5] S. Pulidas, D. Towsley, and J. A. Stankovic, "Embedding gradient estimators in loadbalancing algorithms," Proc. 8th Int. Conf. Distributed Comput. Syst., pp. 482-490,1988.

[6] C. -H. Lee, C. -I. Park, and M. Kim, "Efficient algorithm for graphpartitioning problem using a problem transformation method," Computer-Aided Design, vol. 21, pp. 611-618, Dec. 1989.

[7] V. M. Lo, "Heuristic algorithms for task assignment in distributed systems," IEEE Trans. Comput., vol. C-7-37, pp. 1384-1397, Nov. 1988.

[8] C. -H. Lee, K. G. Shin, "Optimal task assignment in Homogeneous Networks," IEEE Trans. on Parallel and Distributed Systems., vol.8, No.2, pp. 119-129, Feb 1997.

[9] J. -M. Kim, C. -H, Lee, "A Repeated Mapping Scheme of Task Modules with Minimum Communication Cost in Hypercube Multicomputers," ETRI Journal., vol.20, No.4, pp.327-245, Dec, 1998.

[10]A. Salman, I. Ahmad, S. Al-Madani, "Particle swarm optimization for task assignment problem," Microprocessors and Microsystems 26, pp.363-371, 2002.

[11] J. -M.Kim, "Task assignment to consider the communication costs and the load balancing in Distributed and Parallel Systems," PhD thesis., Chungnam National University, Aug. 2003.

[12]B. Ucar, C. Aykanat, K. Kaya and M. Ikinci, "Task assignment in heterogeneous computing systems," j. Parallel Distrib. Comput. 66, pp.32-46, 2006.

[13]J. -M. Orduna, F. Silla and J. Duato, "On the development of a communication-aware task mapping technique,” J. Systems Archit. 50(4), pp.207-220, 2004.

[14]M. Kafil, I. Ahmad, "Optimal Task Assignment in Heterogeneous Distributed Computing Systems," IEEE Concurrency, vol. 6 no. 3, pp.42-51, Jully-Sept. 1998.

[15]A.P. Tom, C.S.R. Murthy, "Optimal task allocation in distributed systems by graph matching and state space search," J. Systems and Software 46 (1) pp.59-75, 1999.

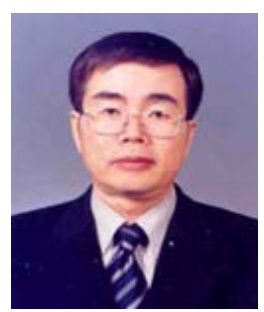

Joo-Man Kim received the B.S. degree in computer science from Soongsil University, Seoul, Korea in 1984 and the M.S. and Ph.D. degrees in computer engineering from Chungnam National University, Daejeon, Korea in 1998 and 2003, respectively. From 1985 to 2000, he worked for ETRI in Daejeon, Korea as principal member for research staff at the OS research team. From 2000 to 2005, he was an assistant professor in the Dept. of Informations and Communications Engineering, Miryang National University. He is currently an associate professor in the Dept. of Applied IT and Engineering, Pusan National University, Pusan, Korea. His research and teaching interests are in embedded system, real-time and ubiquitous computing. 